\title{
Predicting continuum breakdown in hypersonic viscous flows
}

\author{
Wen-Lan Wang ${ }^{\text {a) }}$ and lain D. Boyd ${ }^{\text {b) }}$ \\ Department of Aerospace Engineering, University of Michigan, Ann Arbor, Michigan 48109
}

(Received 21 March 2002; accepted 3 October 2002; published 4 December 2002)

\begin{abstract}
This paper presents a study of the breakdown of the Navier-Stokes equations in hypersonic viscous flows over a sharp cone tip and a hollow cylinder/flare geometry. Investigations are performed through detailed comparisons of the numerical results obtained with continuum and particle techniques. The objective of the study is to predict conditions under which the continuum approach may be expected to fail. A modified breakdown parameter is proposed that can predict the failure of the continuum approach accurately for the simple cone flow and fairly well for the more complex cylinder/flare flow. The study of continuum breakdown is the first step toward development of a hybrid numerical code. (C) 2003 American Institute of Physics. [DOI: 10.1063/1.1524183]
\end{abstract}

\section{INTRODUCTION}

Numerical simulation of hypersonic viscous flows over complex geometries is of great importance because of its application in trans-atmospheric vehicle design. In the flight of such a vehicle, the hypersonic free-stream undergoes large variation in properties due to interactions with shock waves from a wing or control surface and with the boundary layer from the wall surface. The large variation in properties results in some regions where the flow is described as a continuum and can be modeled by the Navier-Stokes (NS) equations and solved numerically by computational fluid dynamics (CFD) approaches. The wide variation in flow properties may also lead to some regions where the flow is rarefied and the NS equations break down because of physical limitations. A particle simulation technique such as direct simulation Monte Carlo ${ }^{1}$ (DSMC) is commonly employed in this region.

The DSMC method cannot be used for the full system, as it demands huge amounts of computational capacity in regions where the flow is dense and in the continuum regime. For instance, in Refs. 2 and 3, computations of the same geometry and free-stream conditions (Run $28^{4}$ ) were performed by CFD and DSMC methods, respectively. The CFD method consumed about 20 hours of 32 processors of an IBM-SP machine and captured all the flow details in good agreement with experiment ${ }^{5}$ whereas the DSMC method spent more than twice the computational time and a lot more memory but satisfactory results still were not reached. With the well-known fact that the NS equations will fail in rarefied flows, it is necessary to have an approach that is physically accurate and numerically efficient. One way to achieve this objective is to combine the DSMC and CFD methods.

There are two primary issues associated with the combination of the two numerical methods. First of all, we need to determine when to switch between the methods. As the NS

\footnotetext{
a) Telephone: 734-764-6573; fax: 734-763-0578. Electronic mail: aerowwl@engin.umich.edu


iainboyd@engin.umich.edu
}

equations are not valid under rarefied conditions, it is general to use a continuum breakdown parameter as the criterion for switching between methods. In his pioneering work, Bird ${ }^{6}$ first advocated a semiempirical parameter for expanding flows. Another empirical parameter based on local flow gradients was later developed specifically for hypersonic flows. ${ }^{7}$ More recently, a new breakdown parameter based on the Chapman-Enskog perturbation expansion of the Boltzmann equation has been developed again for expanding flows. ${ }^{8}$ In the present investigation, the determination of an applicable parameter for hypersonic flows is addressed.

The second issue concerned in a hybrid DSMC-CFD approach is to deal with the information exchange at the interface of the two methods. Several approaches have been considered, such as the Marshak condition, ${ }^{9}$ the KFVS scheme ${ }^{10,11}$ and AMAR embedding a particle method. ${ }^{12}$ Unfortunately, none of these schemes was designed for nonequilibrium, hypersonic compressed flows. Since this issue is beyond the scope of the present research, it will be left for the future study.

The layout of the paper is as follows. A description of two types of breakdown parameter is provided in the next section. We will show the relations between these parameters and propose a modified parameter that is believed to be more conservative and adequate for complex flows. In the section of Numerical Examples, a hypersonic flow over a simple axisymmetric cone tip is first considered, followed by a hypersonic flow over a relatively complex hollow cylinder/flare geometry. In the last section, conclusions and suggestions for future work are provided.

\section{CONTINUUM BREAKDOWN}

It is well known that the NS equations begin to break down under rarefied conditions in which a velocity distribution slightly perturbed from the Maxwellian distribution is difficult to maintain. It is also well known that DSMC is prohibitively expensive for dense flows. An effort is being undertaken to numerically solve flow fields under any conditions by combining CFD and DSMC methods. In general, a computation is initialized by the CFD technique of a hybrid 
approach because of its efficiency. A breakdown criterion is then applied to the intermediate solution, to identify the regions where the physical limitations of the NS equations make the solution invalid. The DSMC technique is called upon to re-evaluate these regions. The hybrid approach will then be applied so that some regions are calculated using DSMC and some using CFD with continuous exchange of information between the two techniques.

The objective of the present investigation is to predict the conditions under which the continuum approach may be expected to fail in hypersonic viscous flows. A breakdown criterion is developed by detailed comparisons of CFD and DSMC solutions.

In prior works of continuum breakdown, Bird $^{6}$ proposed a semiempirical parameter for steady-state expanding flows

$$
P=\frac{U}{\rho \nu}\left|\frac{d \rho}{d s}\right|=M \sqrt{\frac{\pi \gamma}{8}} \frac{\lambda}{\rho}\left|\frac{d \rho}{d s}\right|,
$$

where $U$ is the local velocity, $\nu$ is the collision frequency, $M$ is the local Mach number, $\gamma$ is the ratio of specific heats, and $s$ is the distance along a streamline. Although studies in Ref. 6 indicate the value for $P$ of about 0.05 is a good criterion for continuum breakdown in steady expanding flows, it is believed that in complicated flows density is not the only flow property needed to be taken into account. By the same definition, any flow properties $Q$ (e.g., density, temperature, etc.) can be employed to obtain

$$
P_{Q}=M \sqrt{\frac{\pi \gamma}{8}} \frac{\lambda}{Q}\left|\frac{d Q}{d s}\right| .
$$

Since the evaluation of the gradient in the stream-wise direction involves the velocity components to calculate the breakdown parameter $P_{Q}$, it is generally a problem at stagnation points. Boyd et al. carried out an extensive numerical investigation of one-dimensional normal shock waves and two-dimensional bow shocks comparing DSMC and CFD results, to determine an appropriate breakdown parameter. They concluded that the gradient-length local (GLL) Knudsen number

$$
\mathrm{Kn}_{\mathrm{GLL}}=\frac{\lambda}{Q}\left|\frac{d Q}{d l}\right|,
$$

where $l$ is some distance between two points in the flow field, provides a better indication of continuum breakdown than $P$ for hypersonic compressed flows. They also showed that the distance $l$ should be taken approximately along the line of the steepest gradients in the flow properties. In this study, however, we simply evaluate $d Q / d l$ as $\nabla Q$ without projecting it onto a preferential direction. For simplicity, we remove the subscript GLL from now on and write the GLL Knudsen number for property $Q$ as

$$
\mathrm{Kn}_{Q}=\frac{\lambda}{Q}|\nabla Q|
$$

The Knudsen number of this form has a great physical meaning. When its value is much less than unity the flow can be regarded as locally slightly perturbed from equilibrium ${ }^{13}$ that is a fundamental assumption of the NS equations. Therefore, it is an appropriate parameter to predict continuum breakdown.

It is apparent that Eqs. (1) and (2) are not independent and have the relation

$$
\frac{P_{Q}}{\mathrm{Kn}_{Q}}=M \sqrt{\frac{\pi \gamma}{8}} \cos \theta,
$$

where $\theta$ is the angle between gradient $\nabla Q$ and the flow direction. Since $\sqrt{\pi \gamma / 8}<1$ for most gases, the value of $P_{Q}$ is always less than $\mathrm{Kn}_{Q}$ in subsonic regions. In the region immediately adjacent to the wall surface, because both $M$ and $\cos \theta$ decrease towards zero, $P_{Q}$ is always several orders of magnitude smaller than $\mathrm{Kn}_{Q}$. We will demonstrate the difference numerically in the next section.

Because the breakdown of the NS equations is related to viscosity and heat transfer, we have to take both transport phenomena into account. As a result, density $(D)$, the magnitude of velocity $(V)$, and translational temperature $(T)$ are the flow properties usually considered in the breakdown parameters. We also define a new parameter for $P$ and $\mathrm{Kn}$, respectively,

$$
\begin{aligned}
& P_{\max } \equiv \max \left(P_{D}, P_{T}, P_{V}\right), \\
& \mathrm{Kn}_{\max } \equiv \max \left(\mathrm{Kn}_{D}, \mathrm{Kn}_{T}, \mathrm{Kn}_{V}\right),
\end{aligned}
$$

to be utilized as the actual breakdown parameter for switching between the numerical methods.

\section{NUMERICAL EXAMPLES}

In this study, the NS calculations are performed with an implicit finite-volume CFD code based on the methods discussed in Ref. 2. The fluxes are evaluated with a secondorder accurate flux-vector splitting method based on a modified Steger-Warming method. Viscosity is computed using curve-fits obtained by Blottner et al. ${ }^{14}$ A slip-boundary model proposed by Gökçen ${ }^{15}$ is incorporated.

The particular DSMC code, named MONACO, employed in this study was first developed by Dietrich and Boyd. ${ }^{16}$ MONACO employs the variable soft sphere (VSS) collision model, ${ }^{17}$ the variable rotational energy exchange probability model of Boyd ${ }^{18}$ and the variable vibrational energy exchange probability model of Vijayakumar et al. ${ }^{19}$ Cell weighting factors and time-steps may be set uniquely for each cell in the grid. A sub-cell scheme is implemented for selection of collision pairs where the number of sub-cells is scaled by the local mean free path.

The numerical examples considered in this paper refer to the experiments performed in CUBRC impulse test facilities (see Ref. 4). The fluid is pure nitrogen and the free-stream conditions are listed in Table I. The wall temperature $T_{w}$ of both examples is assumed constant in the computations.

\section{A. Run 35}

The original configuration of CUBRC Run 35 is a sharp double cone with half angles of $25^{\circ}$ and $55^{\circ}$. Because it is difficult for DSMC to reach the steady state solution under 
TABLE I. Free stream conditions of CUBRC experiments.

\begin{tabular}{lcc}
\hline \hline & Run 35 & Run 11 \\
\hline$M_{\infty}$ & 11.3 & 11.3 \\
$T_{\infty}(\mathrm{K})$ & 138.9 & 128.9 \\
$V_{\infty}(\mathrm{m} / \mathrm{s})$ & 2712.2 & 2609.1 \\
$\rho_{\infty}\left(10^{-3} \mathrm{~kg} / \mathrm{m}^{3}\right)$ & 0.552 & 0.507 \\
$\operatorname{Re}_{L}\left(10^{3}\right)$ & 14.5 & 13.8 \\
$T_{w}(\mathrm{~K})$ & 296.1 & 297.2 \\
\hline \hline
\end{tabular}

the specific free-stream conditions for the full configuration, ${ }^{3}$ consideration is given only to the cone tip of the forecone.

In the present study, the DSMC computation employs a structured grid with 1200 cells along the cone surface by 400 cells normal to the cone. A reference time step of $0.5 \mathrm{~ns}$ is used. More than 8 million simulation particles are employed at the end of the computation that consumes a total of 82 hours on 16 processors on an IBM-SP machine. The results presented below are obtained by sampling over 50000 time steps.

Comparisons of the density and translational temperature contours obtained with CFD and DSMC are made in Figs. 1(a) and 1(b), respectively. Good agreement is shown in general between the two solutions.

A detailed comparison of the flow properties obtained with the NS and DSMC approaches is made in Fig. 2(a) at $x / L=0.1$ [dotted line $\mathrm{b}$ in Fig. 1(a)], where $x$ is the axial distance from the leading edge of the cone tip and $L$ $=92.07 \mathrm{~mm}$ is the length of the forecone. In this figure, $\delta n$ is the distance from the cone surface and $\epsilon_{Q}$ defined as

$$
\epsilon_{Q} \equiv \frac{Q_{\mathrm{CFD}}}{Q_{\mathrm{DSMC}}}-1,
$$

represents the degree of difference between the NS and DSMC solutions. Throughout this paper, for the purposes of discussion, failure of the continuum approach is defined to occur whenever $\left|\epsilon_{Q}\right|>0.05$. It is shown in this figure that the DSMC shock is much thicker than the CFD shock, suggesting strong nonequilibrium effects. The thicker DSMC shock results in the negative $\epsilon$ for density and temperature ahead of the CFD shock. Near the cone surface, the substantial difference is assumed to indicate the failure of the NS approach. In particular, the NS density close to the surface is about $70 \%$ higher than the DSMC solution. In contrast, the NS velocity and temperature are, respectively, $40 \%$ and $90 \%$ lower than the DSMC solutions, indicating a prediction of less slip by the model employed in CFD. In between the shock and nearbody regions, the solutions of the CFD and DSMC techniques agree approximately.

Profiles of $P$ and Kn based on the NS solutions are shown in Fig. 2(b). A 0.05 line is also shown in the figure to indicate the breakdown of the NS approach. All breakdown parameters vanish in the free-stream and increase rapidly across the shock wave. As discussed above, the continuum approach fails in the region immediately adjacent to the wall surface. It is clear that the $\mathrm{Kn}$ parameters capture the breakdown very well in this region. In other words, the hybrid code will successfully detect the continuum breakdown and
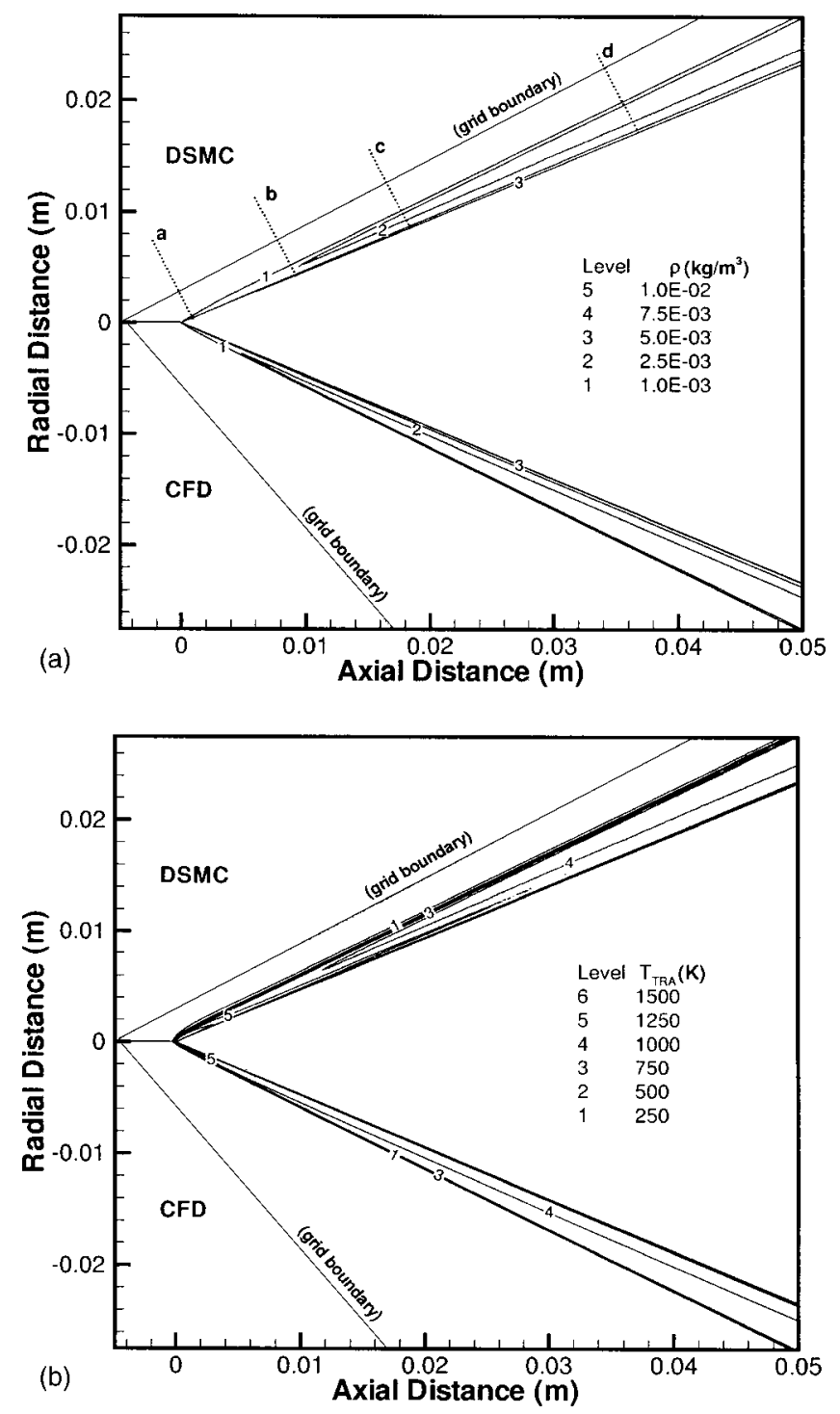

FIG. 1. Comparison of DSMC and CFD solutions of (a) density and (b) translational temperature.

prepare to switch to the DSMC method. By comparison, the $P$ parameters are not able to predict breakdown close to the wall since these values become very small. This is attributed to the small value of the Mach number and approximate right angle between the flow and gradient directions.

Similarly, profiles of $P$ and Kn based on the DSMC solutions are illustrated in Fig. 2(c). The profiles qualitatively agree with the profiles in Fig. 2(b) in the region close to the body surface. The peak of each DSMC parameter across the shock is lower than the corresponding value of the CFD results in Fig. 2(b) because of weaker gradients over the thicker shock in the DSMC solutions.

In Figs. 2(b) and 2(c), one can observe that $\mathrm{Kn}_{D}$ and $\mathrm{Kn}_{T}$ are almost identical near the wall. A proof of this observation is given in the Appendix.

It is shown in Fig. 2(a) over $0<\delta n<0.38$, that $\left|\epsilon_{T}\right|$ $>0.05$ and in Figs. 2(b) and 2(c) $\mathrm{Kn}_{V}$ is the only parameter that can predict the continuum breakdown in this region. However, $\mathrm{Kn}_{V}$ does not accurately predict the continuum breakdown at the shock. At about $\delta n=1.15, \epsilon_{T}$ is approxi- 

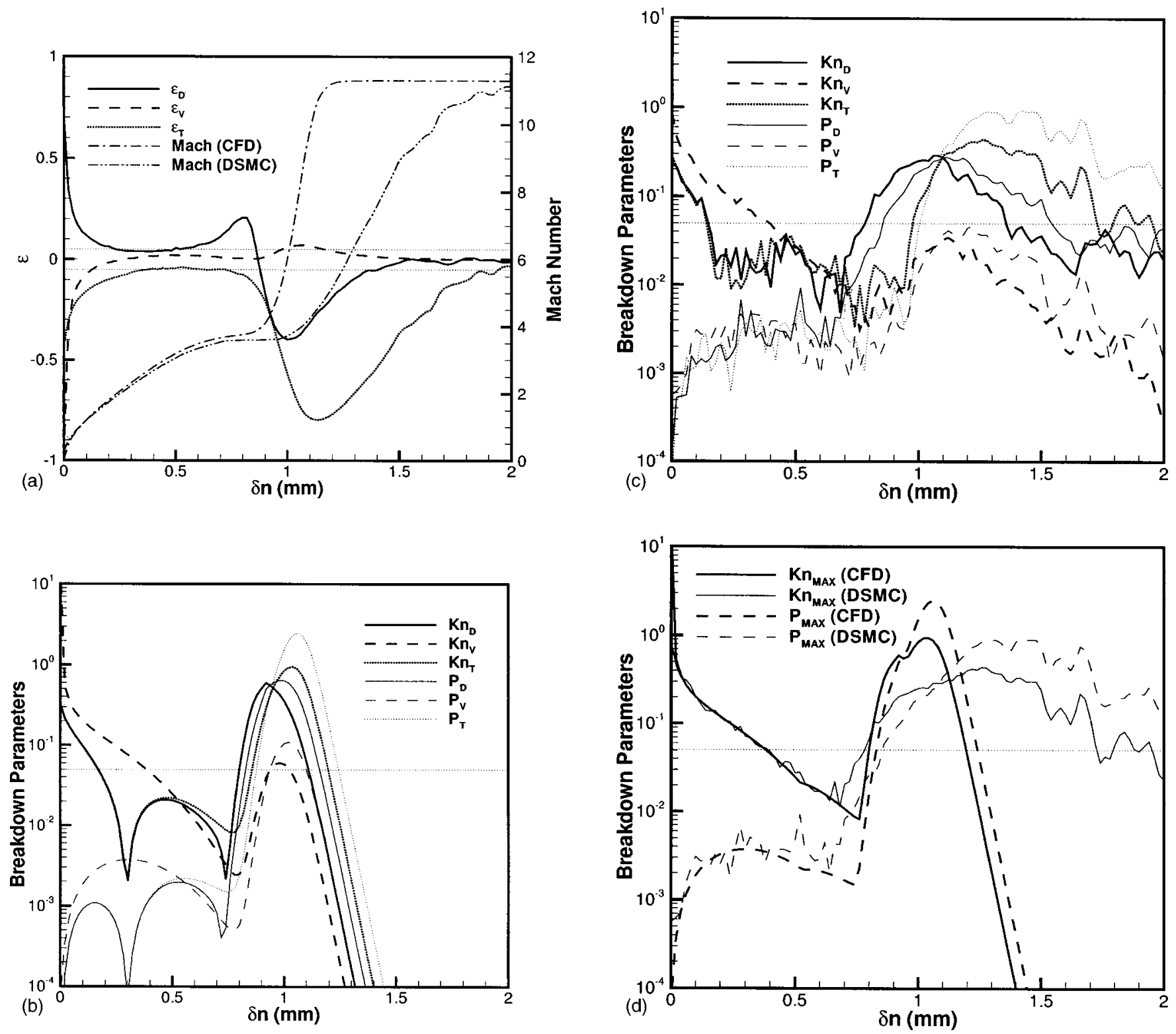

FIG. 2. Profiles along the line normal to the cone surface at $x / L=0.1$. (a) Comparison of density, velocity, temperature, and Mach number. (b) Breakdown parameters based on NS solutions. (c) Breakdown parameters based on DSMC solutions. (d) Variation of $\mathrm{Kn}_{\max }$ and $P_{\max }$ based on NS and DSMC solutions.

mately -0.8 . At the same location, $\mathrm{Kn}_{V}$ is about 0.006 and 0.03 for the CFD and DSMC solutions, respectively, both below 0.05 . In comparison, $\mathrm{Kn}_{T}$ reaches about 0.2 for both solutions. We conclude that it is necessary to evaluate $\mathrm{Kn}$ based on various flow properties at each location in order to predict the continuum breakdown accurately.

In Fig. 2(d) profiles of $P_{\text {max }}$ and $\mathrm{Kn}_{\max }$ defined in Eqs. (4) and (5) are displayed. It is clear that near the body surface, $\mathrm{Kn}_{\max }$ evaluated with the CFD and DSMC results are very close and predict continuum breakdown whereas $P_{\max }$ are much lower than 0.05. In comparison of Figs. 2(a) and 2(d), one can find that use of $\mathrm{Kn}_{\max }$ to predict the failure of the continuum approach is quite successful. In the region of $\delta n$ from 0 to about 0.38 , Fig. 2(d) has values of $\mathrm{Kn}_{\max }$ higher than 0.05 and Fig. 2(a) shows the corresponding continuum breakdown. From $\delta n \sim 0.38$ to $\delta n \sim 0.8, \mathrm{Kn}_{\max }$ evaluated with the CFD solution is below the criterion and flow prop- erties from the two methods agree except for a small fraction of the post-shock region. For the rest of the region from $\delta n \sim 0.8$ to the freestream boundary, $\mathrm{Kn}_{\max }$ calculated with the DSMC solution is higher than the criterion in general and continuum breakdown is observed in Fig. 2(a).

In Fig. 3(a), the NS and DSMC solutions are compared in detail along a normal line to the cone at $x / L=0.2$ [dotted line $\mathrm{c}$ in Fig. 1(a)]. It is evident that the differences of the two solutions at this station are similar to those in Fig. 2(a). The $\mathrm{Kn}_{\max }$ and $P_{\max }$ values based on the CFD and DSMC solutions are illustrated in Fig. 3(b). The continuum breakdown near the wall of the cone is predicted by $\mathrm{Kn}_{\max }$ but not by $P_{\max }$. One can find the same CFD/DSMC switch as discussed above. Similarly, the profiles of comparison and breakdown parameters are displayed in Fig. 4 along the line normal to the cone at $x / L=0.4$ [dotted line d in Fig. 1(a)].

One can find in Figs. 2-4 through that although $\mathrm{Kn}_{\max }$ 

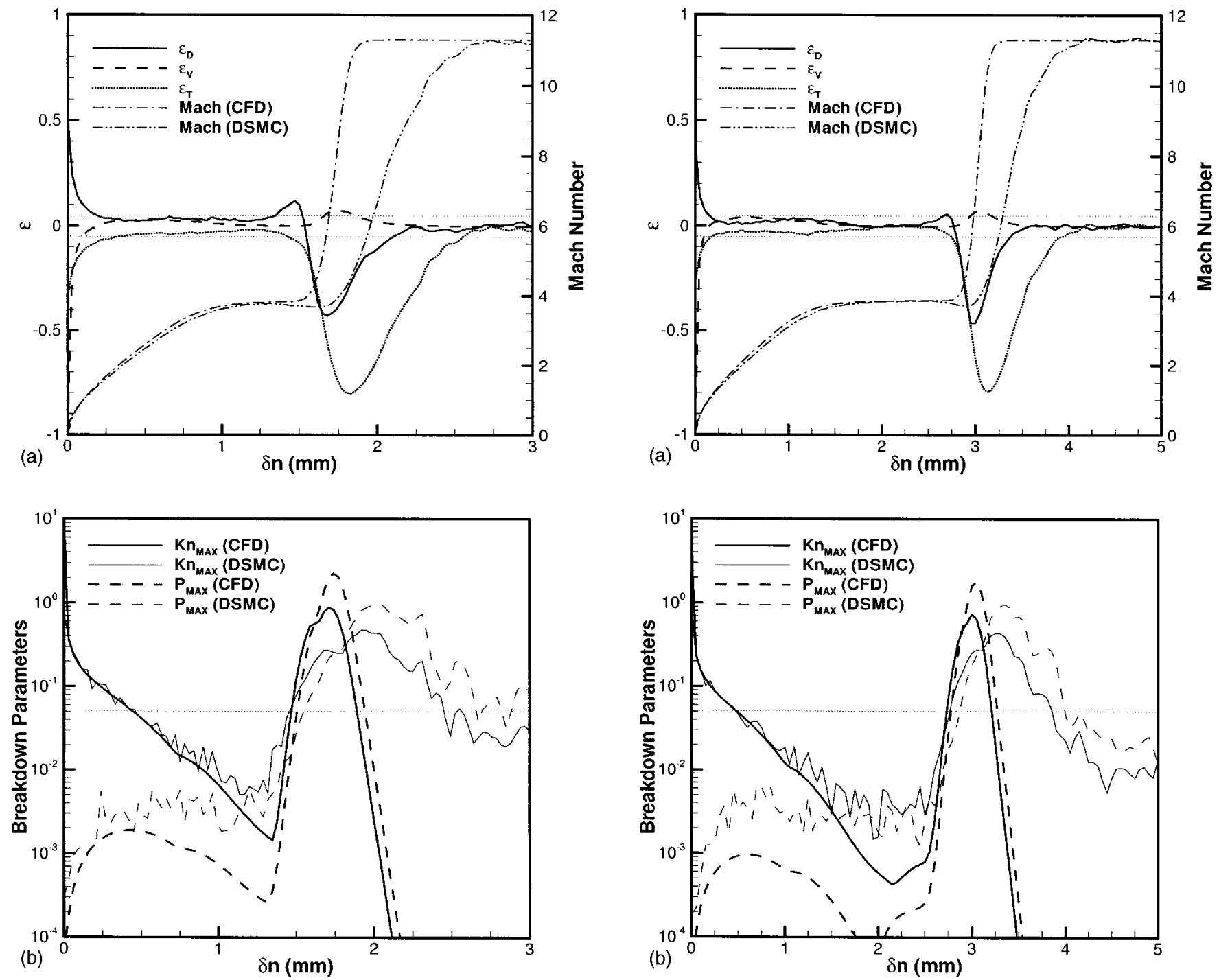

FIG. 3. Profiles along the line normal to the cone surface at $x / L=0.2$. (a) Comparison of density, velocity, temperature, and Mach number. (b) Variation of $\mathrm{Kn}$ and $P$ based on NS and DSMC solutions.

has not demonstrated the capability of prediction of the failure of the continuum approach in some regions, especially in the post-shock region, this is not a serious concern. Keep in mind the comparisons are made of the steady-state solutions obtained with two different numerical techniques. The flow details, such as the shock angle and thickness, are not identical in the two solutions. As a result, significant differences in the post-shock region were expected. Our goal is that the differences between the DSMC and the hybrid code solutions in the future shall lie within the $\pm 5 \%$ band and $\mathrm{Kn}$ based on the hybrid code solutions shall completely predict all breakdown.

Strong thermal nonequilibrium near the leading edge of the cone has been reported in Ref. 20. The profiles of the comparisons between the NS and DSMC solutions for the flow properties near the leading edge at $x / L=0.01$ [dotted line a in Fig. 1(a)] are shown in Fig. 5(a). Clearly, the shock angle and thickness calculated with the CFD technique do not agree with the DSMC results. The rarefaction

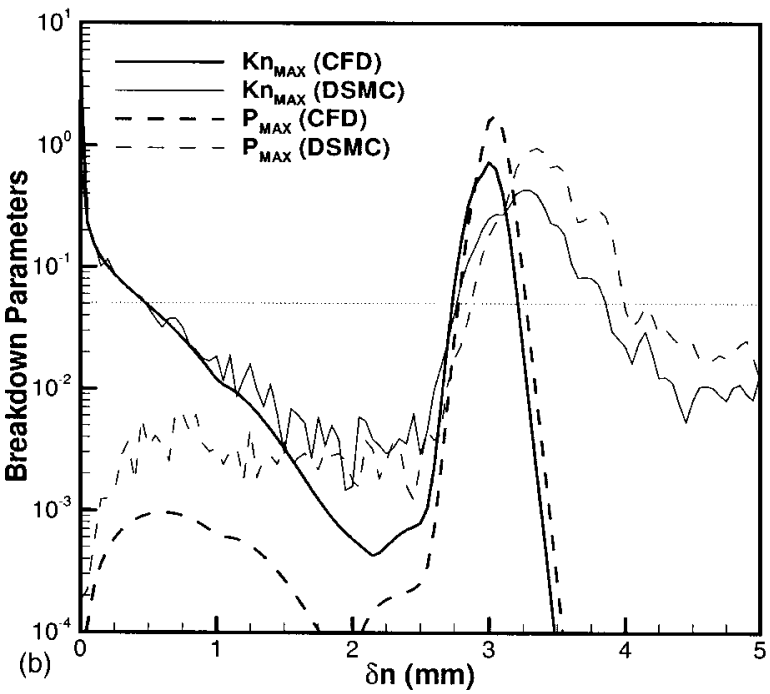

FIG. 4. Profiles along the line normal to the cone surface at $x / L=0.4$. (a) Comparison of density, velocity, temperature, and Mach number. (b) Variation of Kn and $P$ based on NS and DSMC solutions.

parameter, ${ }^{21} \bar{V}$, is evaluated locally as about 0.74 . This value is about 5 times higher than the upper limit, $\bar{V} \approx 0.15$, for the Rankine-Hugoniot shock structure theory to be valid. The accuracy of the NS approach at this station must be consequently poor. Note the mix of the post-shock region with the boundary layer, which indicates interactions between the two regions. $\mathrm{Kn}_{\max }$ based on the DSMC solutions is higher than the criterion over the entire region considered [see Fig. 5(b)]. This suggests the use of the DSMC approach near the leading edge is necessary.

It is interesting to point out that the failure of the NS equations near the leading edge should have a connection with the continuum breakdown in the rest of the flow field. One can imagine that the continuum breakdown from the shock and near-body in the region close to the leading edge is mixed together and separates gradually in the downstream. One branch follows the path of the shock, which departs from the cone surface. The other branch stays in the region immediately adjacent to the wall. By the same token, some 

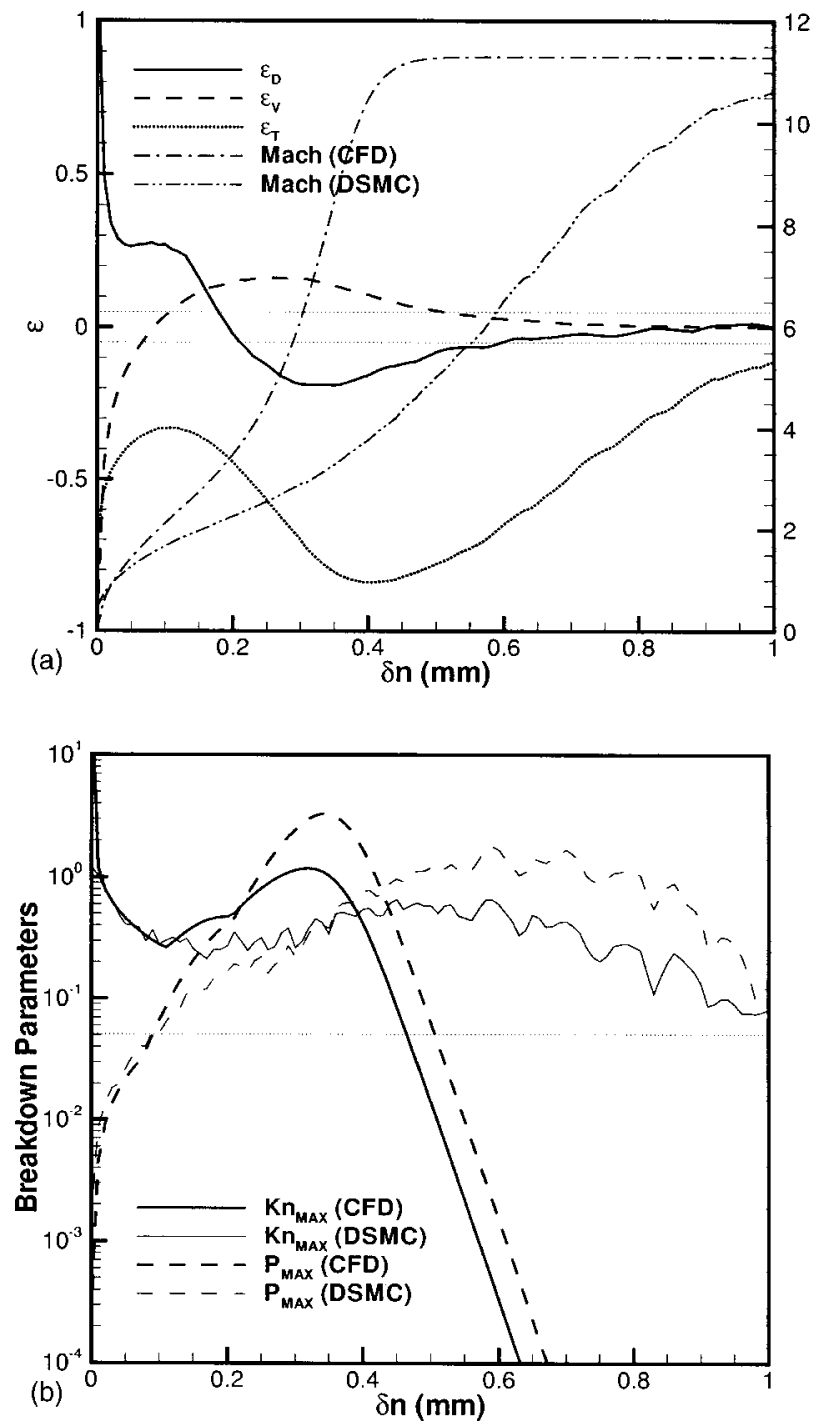

FIG. 5. Profiles along the line normal to the cone surface at $x / L=0.01$. (a) Comparison of density, velocity, temperature, and Mach number. (b) Variation of $\mathrm{Kn}$ and $P$ based on NS and DSMC solutions.

of the differences between NS and DSMC for large $x / L$ are due to differences that first occur at the leading edge and then propagate.

The results shown in Figs. 2-5 for the hypersonic flow over a sharp $25^{\circ}$ half-angle cone tip may be summarized as follows. Near the body surface, the steep flow gradients cause the continuum equations to fail and to predict the flow properties incorrectly. This has a great impact for the continuum estimation of surface values such as heat transfer rate and pressure. Accordingly, the DSMC technique must be employed in this region. Moving away from the body, there is a region where the solutions of the NS and DSMC approaches agree approximately. The NS method should be used in this region. Moving further away from the body and approaching the shock where the flow gradients are steep and the continuum equations break down again, the flow should be calculated using the DSMC method from here out to the freestream boundary. $\mathrm{Kn}_{\max }$ is found to be quite successful for the prediction of the breakdown using a value of 0.05 for the criterion for switching between the numerical methods.

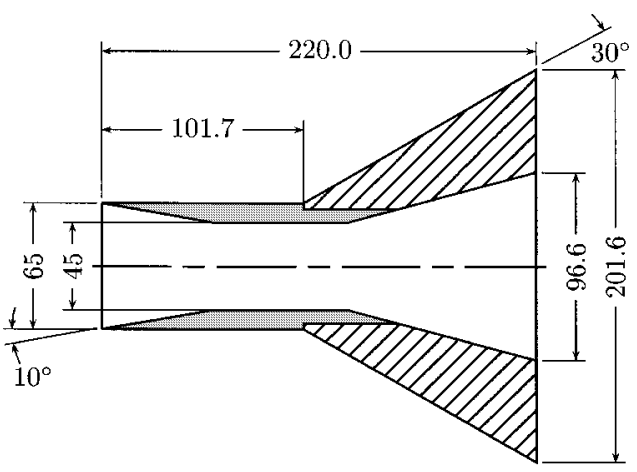

FIG. 6. Schematic of the CUBRC hollow cylinder/flare configuration (measurements in $\mathrm{mm}$ )

\section{B. Run 11}

The configuration of CUBRC Run 11 consists of a hollow cylinder followed by a $30^{\circ}$ conical flare, as depicted in Fig. 6. The cylinder is aligned with the free stream. The
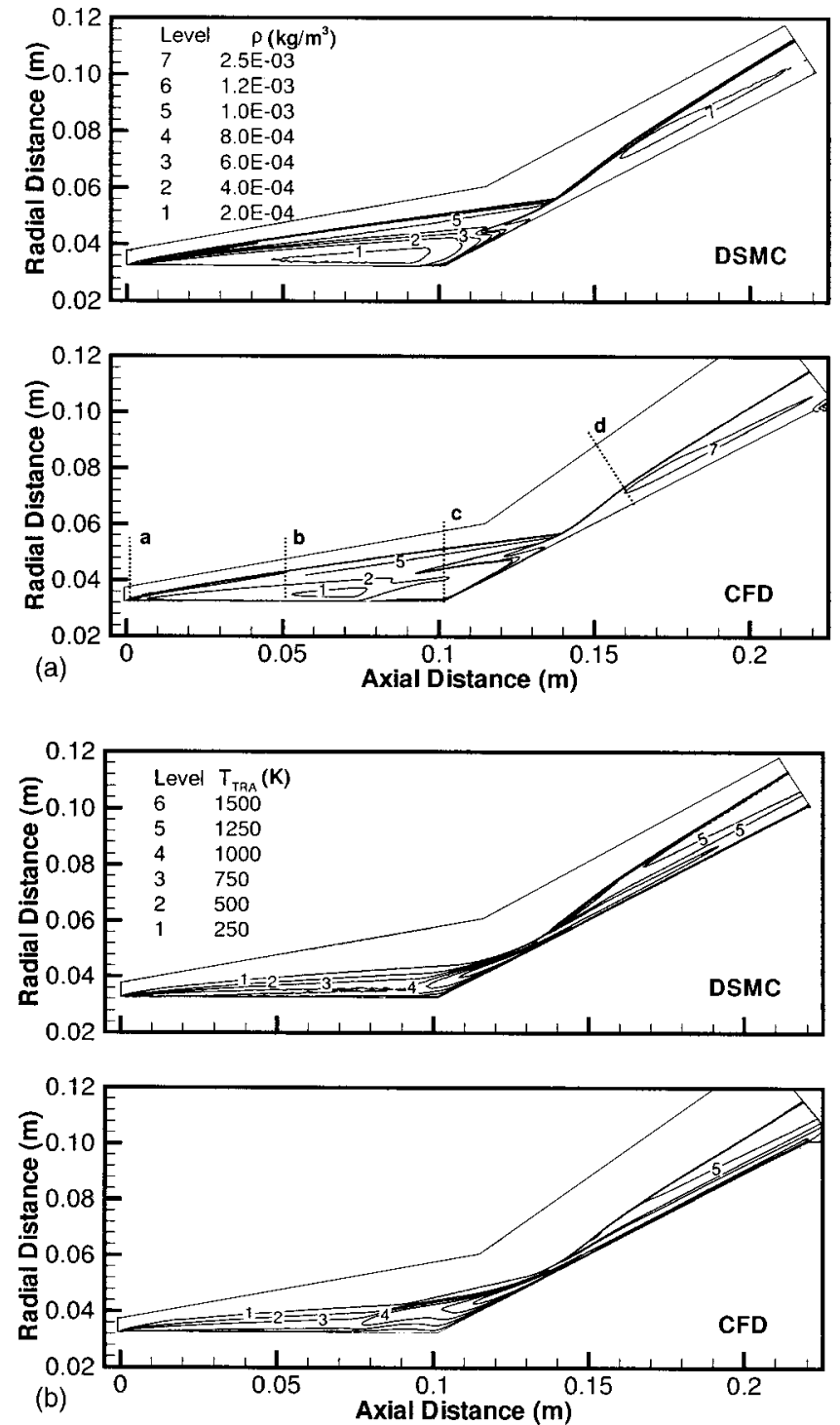

FIG. 7. Comparison of DSMC and CFD solutions of (a) density and (b) translational temperature. 

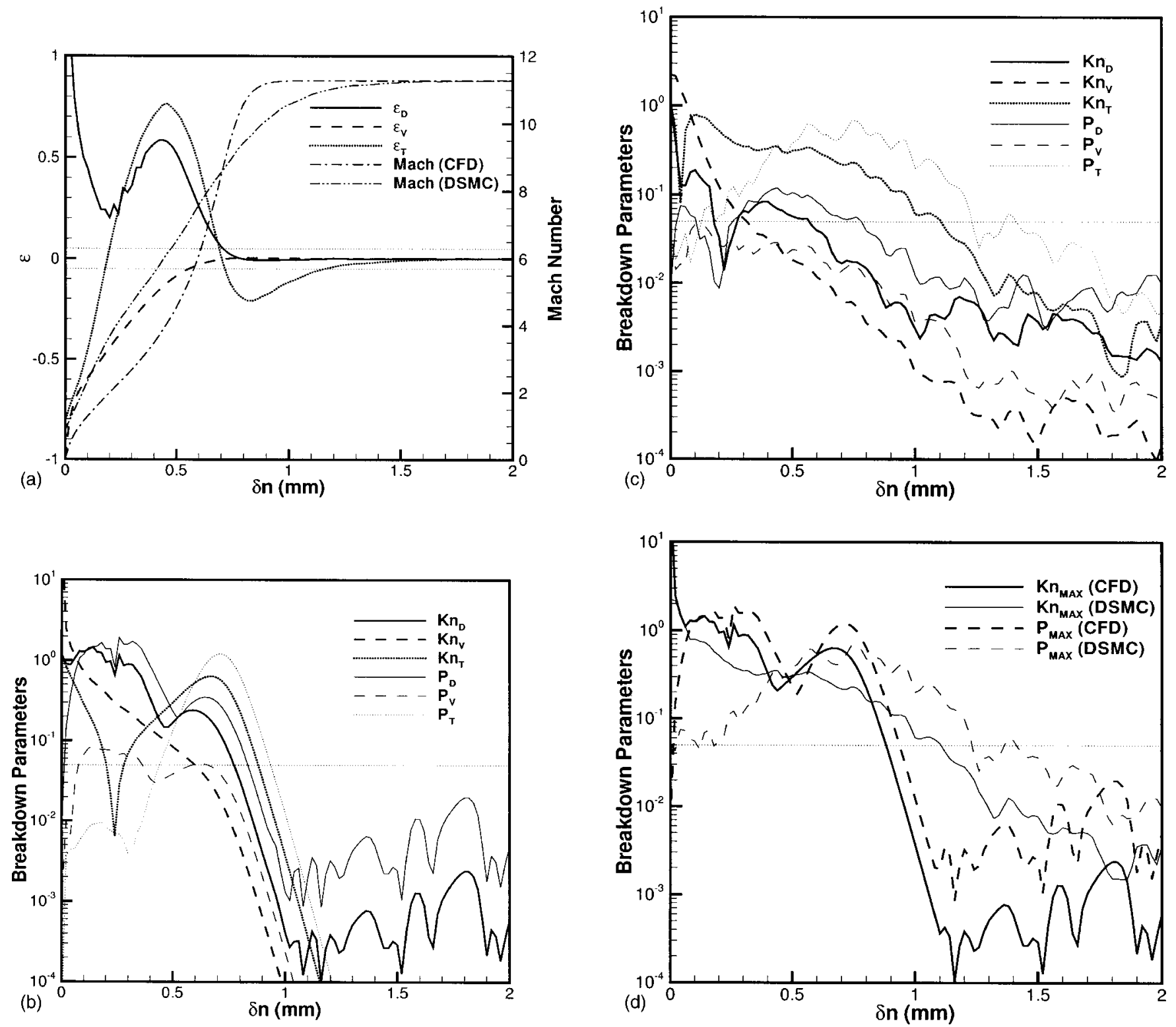

FIG. 8. Profiles along the line normal to the cylinder surface at $x / L=0.01$. (a) Comparison of density, velocity, temperature, and Mach number. (b) Breakdown parameters based on NS solutions. (c) Breakdown parameters based on DSMC solutions. (d) Variation of $\mathrm{Kn}_{\max }$ and $P_{\max }$ based on NS and DSMC solutions.

leading edge is sharp and the hypersonic flow entering the hollow body does not interact with the external flow. We focus only on the external flow.

The DSMC computation employs 1000 by 200 cells with a total of more than 3.5 million simulation particles. The reference time step of this computation is $5 \mathrm{~ns} .800000$ time steps are computed on an IBM-SP machine to consume a total of about 176 hours on 16 processors and the last 100000 time steps are sampled to obtain the results presented below.

In Figs. 7(a) and 7(b) comparisons of the density and transnational temperature contours obtained with CFD and DSMC are shown. A general impression of the complex flow is the gas density is reduced by a factor of about 3 from the free-stream value in the region above the cylinder and then compressed more than 5 times by the flare. The temperature above the cylinder is increased due to viscous interaction and reaches a peak value of about $1000 \mathrm{~K}$. The strong compression caused by the flare leads to further heating with a peak value of approximately $1500 \mathrm{~K}$. Detailed numerical studies of this flow can be found in Refs. 2, 3, and 5. One can notice the significant differences between the CFD and DSMC solutions around the compression corner. There is a larger separation and re-attachment region in the CFD solutions.

To study the continuum breakdown near the leading edge of the hollow cylinder in this case, comparisons for density, velocity, and transnational temperature along the line normal to the body at $x / L=0.01$ [dotted line a in Fig. 7(a)] are made in Fig. 8(a), where $x$ is measured from the leading edge of the hollow cylinder and $L=101.7 \mathrm{~mm}$ is the length of the hollow cylinder. Since there are strong interactions between shock and viscous effects near the leading edge, the post-shock flow quickly merges with the boundary layer. Continuum breakdown is expected, as shown in the figure. 

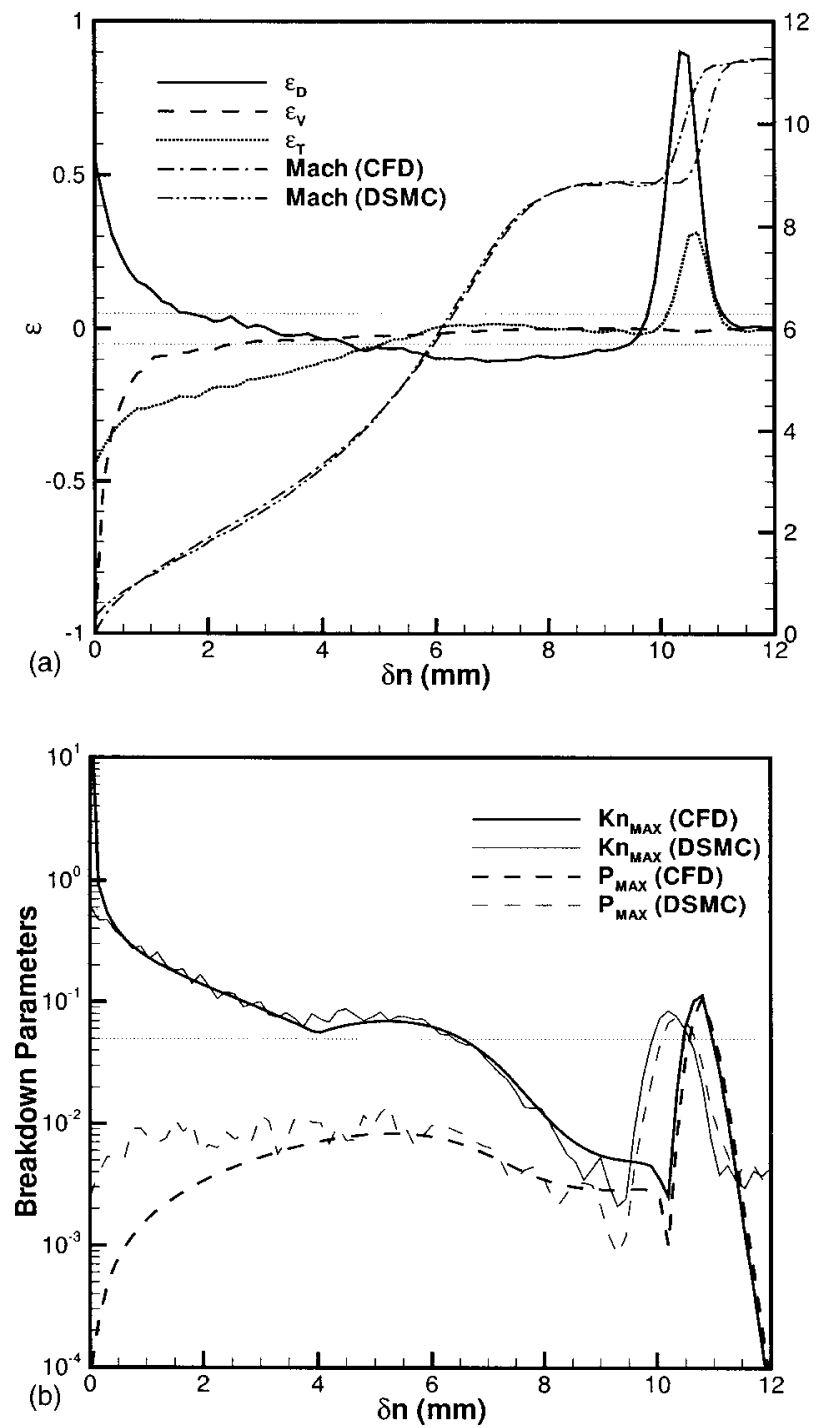

FIG. 9. Profiles along the line normal to the cylinder surface at $x / L=0.5$. (a) Comparison of density, velocity, temperature, and Mach number. (b) Variation of Kn and $P$ based on NS and DSMC solutions.

The breakdown occurs from $\delta n=0$ to about $1.1 \mathrm{~mm}$. Breakdown parameters evaluated with various flow properties based on the CFD solution are displayed in Fig. 8(b). The value of 0.05 is again chosen to be the criterion. Like the previous cone tip case, the Kn parameters predict the failure of the continuum approach at the body surface but the $P$ parameters do not. The profiles of $\mathrm{Kn}$ and $P$ calculated using the DSMC solution are in Fig. 8(c). Again, the Kn's can capture the continuum breakdown but the $P$ 's do not. Focus on the $\mathrm{Kn}_{\max }$ and $P_{\text {max }}$ that are shown in Fig. 8(d). Keeping in mind that the DSMC technique must be applied in the region near the body surface, as discussed above, one can find that $\mathrm{Kn}_{\max }$ based on DSMC crosses the 0.05 criterion line at about $1.1 \mathrm{~mm}$ which is the boundary of the continuum breakdown. Other parameters either underestimate the breakdown range or fail to predict the breakdown in the region very close to the body.

Next, the middle of the cylinder is studied, where the shock and boundary layers separate. In Fig. 9 profiles along the dotted line b in Fig. 7(a) are shown of comparisons for
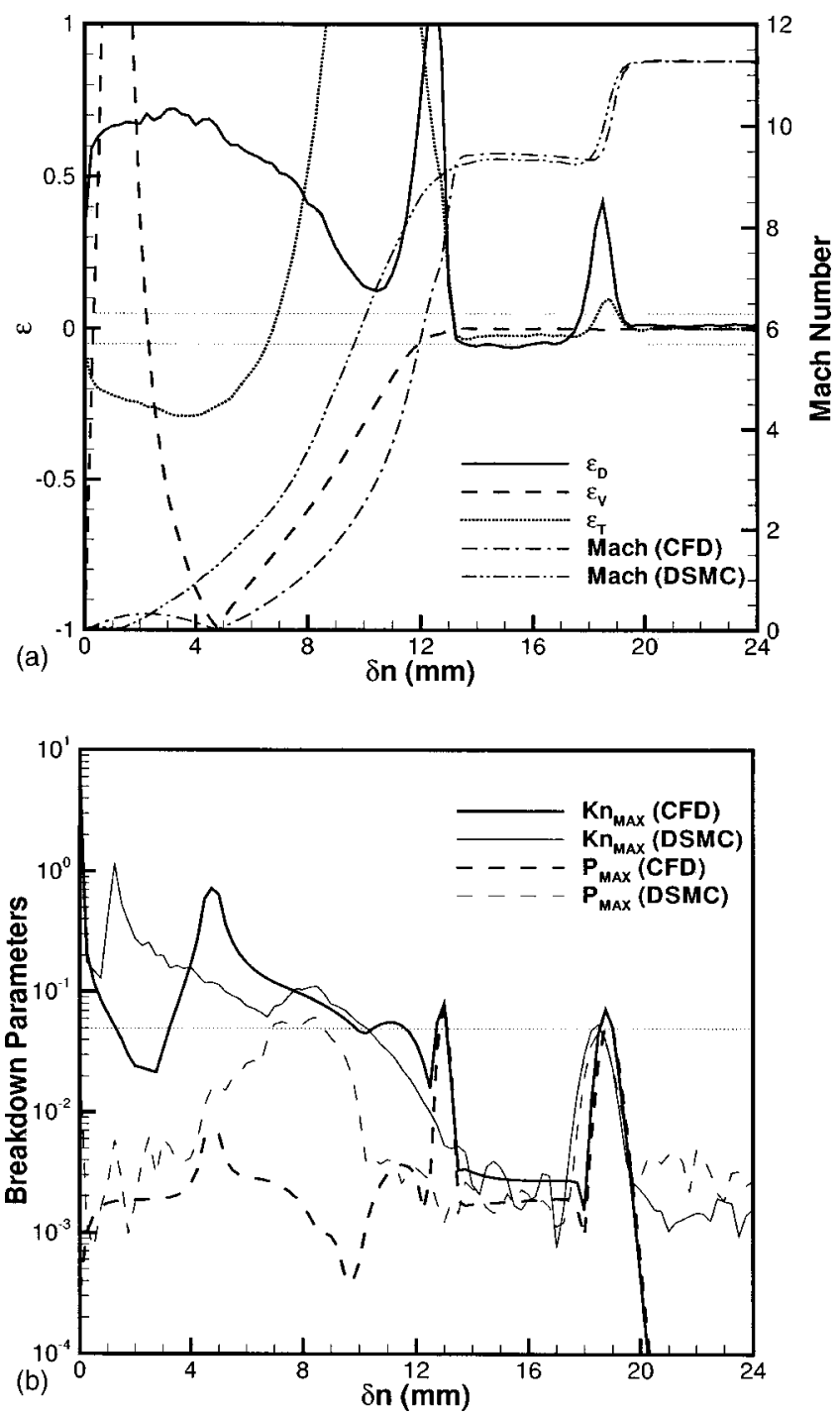

FIG. 10. Profiles along the line normal to the cylinder surface at $x / L$ $=1.0$. (a) Comparison of density, velocity, temperature, and Mach number. (b) Variation of $\mathrm{Kn}$ and $P$ based on NS and DSMC solutions.

the flow properties considered and the breakdown parameters. It is noticed that the CFD shock is a little further from the wall than the DSMC shock at this station but their thicknesses are about the same. In the range of $\delta n$ shown, there is not a single place where all the $\epsilon_{Q}$ considered lie within the $\pm 5 \%$ band, except in the free stream. This is in part because of different shock locations obtained with the DSMC and CFD methods. More importantly, it is also in part because of the strong continuum breakdown from the steep flow gradients near the body. This can be verified in Fig. 9(b) in which the values of $\mathrm{Kn}_{\max }$ based on both CFD and DSMC solutions are above the criterion line until about $6.5 \mathrm{~mm}$. Although it is not a precise prediction for the range of the continuum breakdown, it is indeed an indication of $\mathrm{Kn}_{\max }$ being promising for prediction of the continuum breakdown in complex flows.

As mentioned in the beginning of this subsection, the flow structures obtained with the CFD and DSMC approaches are quite different around the junction of the cylinder and the flare. Flow properties along the dotted line $\mathrm{c}$ in 

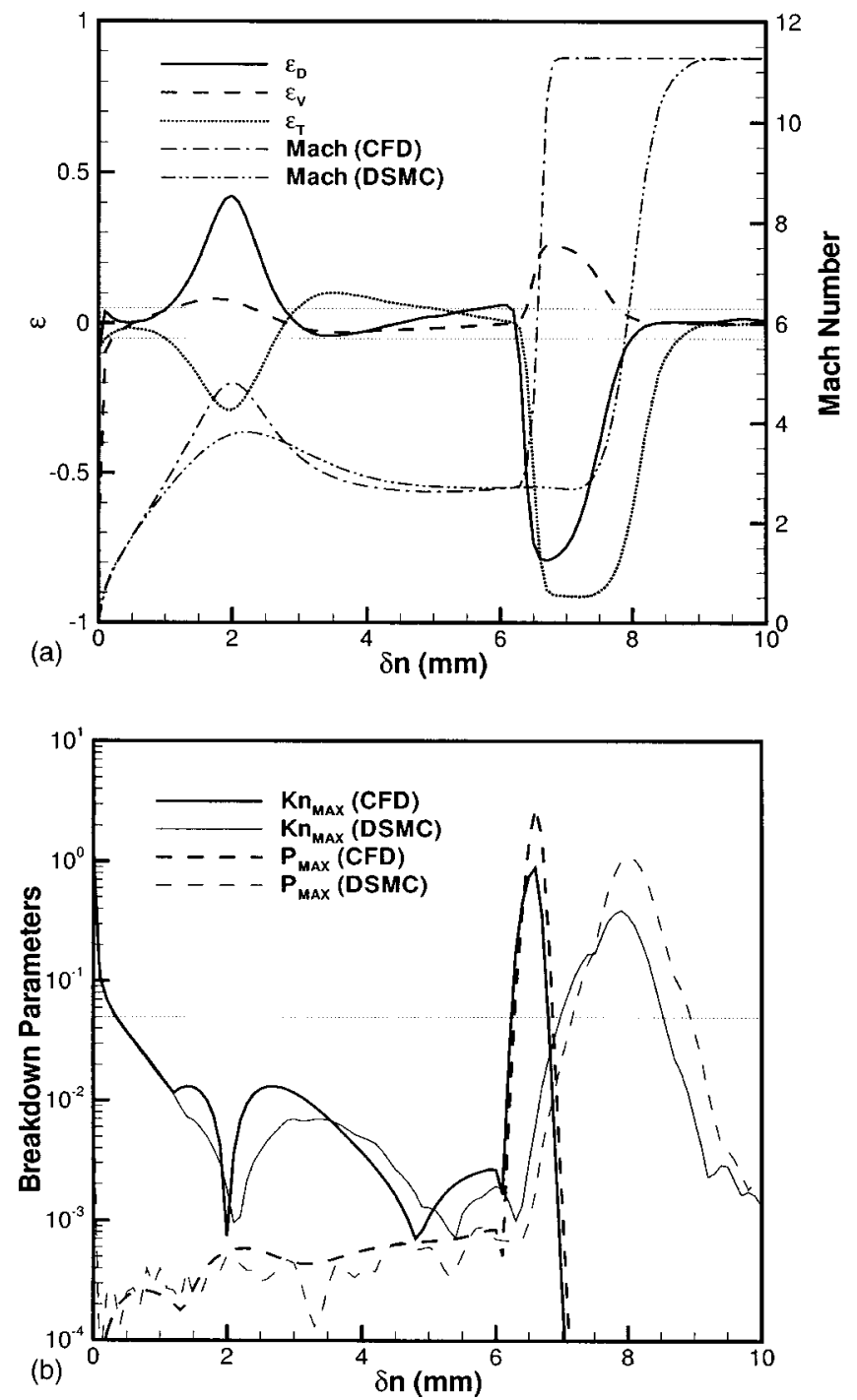

FIG. 11. Profiles along the line normal to the flare surface at $x / L=1.6$. (a) Comparison of density, velocity, temperature, and Mach number. (b) Variation of $\mathrm{Kn}$ and $P$ based on NS and DSMC solutions.

Fig. 7(a) are shown in Fig. 10(a). The profiles of Mach number for the CFD and DSMC solutions in the boundary layer are inconsistent to a large extent. Attention should be paid to the lower left corner of the figure where each Mach number profile shows two stagnation points, one is right at the junction and the other is in the circulation zone. The second stagnation point is at about $5 \mathrm{~mm}$ for the CFD method and about $1.5 \mathrm{~mm}$ for DSMC above the body. One can also find a huge value of $\epsilon_{V}$ at about $1.5 \mathrm{~mm}$. This is because $Q_{\mathrm{DSMC}}$ in Eq. (6) is very small at a stagnation point.

The profiles of $\mathrm{Kn}_{\max }$ and $P_{\max }$ at the station are shown in Fig. 10(b). The $\mathrm{Kn}_{\max }$ evaluated with the DSMC solution is still promising to predict the breakdown at the wall and covers the major part of the region where the NS equations fail. At the shock front, the $\mathrm{Kn}_{\max }$ based on the CFD solution once again predicts the breakdown.

Finally, a station on the flare [dotted line d in Fig. 7(a)] is examined where the free stream crosses a strong oblique shock [see Fig. 11(a)]. The solutions of the CFD and DSMC methods have a large discrepancy in most regions, except for a narrow region close to the flare surface. As shown in Fig. 11(b), $\mathrm{Kn}_{\max }$ successfully captures the breakdown on the wall but not in most regions of the boundary layer. On the other hand, $P_{\max }$ completely fails to predict the breakdown of the NS equations in the boundary layer. Both $\mathrm{Kn}_{\max }$ and $P_{\text {max }}$ predict very well the breakdown across the shock.

Analogous to the first example, the hypersonic flow over the hollow cylinder/flare configuration of CUBRC Run 11 are not described accurately using the NS equations in the regions near the body surface and the shock and the DSMC technique has to be used. Upstream of the shock, the CFD method will be employed. It is not very clear at this point exactly which method should be used in between the shock and the near-body. The answer will not be available until the hybrid code is developed.

\section{CONCLUDING REMARKS}

A numerical study has been conducted for hypersonic nitrogen flows over an axisymmetric sharp cone tip and a hollow cylinder/flare configuration. The focus of the current study was to identify a criterion that can successfully predict the conditions under which the continuum Navier-Stokes equations may be expected to fail in the hypersonic flows considered. Investigation was carried out by the detailed comparisons of numerical solutions obtained with CFD and DSMC techniques.

A new parameter, $\mathrm{Kn}_{\max }$ is proposed that is modified from Boyd's $\mathrm{Kn}_{\mathrm{GLL}}$ parameter. It is concluded that continuum breakdown is best predicted wherever the value of $\mathrm{Kn}_{\max }$ exceeds 0.05 . For the simple sharp cone flow, the $\mathrm{Kn}_{\max }$ parameter can predict the failure of the continuum approach accurately in terms of the positions of significant differences between the CFD and DSMC solutions, including the regions immediately adjacent to the cone surface and at the shock front. For the more complex hollow cylinder/flare flow, the parameter works fairly well to predict the continuum breakdown at the shock front and at the body surface. In general, $\mathrm{Kn}_{\max }$ does not successfully capture the breakdown in the boundary layer. Since the detailed flow structures of the CFD and DSMC results for this complex flow do not agree to some extent in the boundary layer, it is not possible to conclude that $\mathrm{Kn}_{\max }$ will work in this region in the hybrid code.

It should be pointed out that $\mathrm{Kn}_{\max }$ is still an empirical parameter. The manner in which this empirical parameter is capable of predicting the continuum breakdown in the two test cases does not necessarily mean it will work well for other flows.

When the $\mathrm{Kn}_{\max }$ parameter is utilized in the coupled DSMC-CFD computations, it is expected that the entire flow field will be approximately separated into four layers. In the region very close to the body surface, the DSMC technique will be employed. In between the near-body region and the shock region, the CFD technique will be used. The DSMC technique will be employed again throughout the shock. In the free-stream region, it is efficient to utilize the CFD technique. 
At the interface between the DSMC and CFD regions, macroscopic flow properties have to be provided to the CFD method to evaluate the net fluxes and to the DSMC method to initialize the particles entering from the continuum region into the rarefaction region. The DSMC method always has to employ a relatively small time step to meet its basic assumptions and to sample a period of time to provide smooth macroscopic flow properties. Consequently, the time steps used in the CFD and DSMC techniques are different. An algorithm to filter out the scatter from the DSMC results is also necessary. In addition, it has been shown ${ }^{9,10}$ that initializing particles with the Maxwellian distribution is unacceptable in a particle method coupled with a Navier-Stokes solver and the Chapman-Enskog distribution must be employed. The framework of the development towards a hybrid DSMCCFD approach involves the integration of these issues.

\section{ACKNOWLEDGMENTS}

The authors gratefully acknowledge the efforts of Graham Candler and Ioannis Nompelis for providing the CFD results. The views and conclusions contained herein are those of the authors and should not be interpreted as necessarily representing the official policies or endorsements, either expressed or implied, of the AFOSR or the U.S. Government. The authors would also like to express thanks for the assistance of the Minnesota Supercomputing Institute. This work was sponsored by the Army Research Office under Grant No. DAAG55-98-1-0500 and by the Air Force Office of Scientific Research under Grant No. F49620-01-10003.

\section{APPENDIX: COMPARISON OF BREAKDOWN PARAMETERS}

By taking the ratio of the two Knudsen numbers

$$
\frac{\mathrm{Kn}_{D}}{\mathrm{Kn}_{T}}=\frac{T}{\rho}\left|\frac{\nabla \rho}{\nabla T}\right|,
$$

and recognizing in the boundary layer that the derivative in the normal direction $n$ is much larger in magnitude than in the tangential direction, the ratio can be approximated as

$$
\frac{\mathrm{Kn}_{D}}{\mathrm{Kn}_{T}} \approx \frac{T}{\rho}\left|\frac{\partial \rho / \partial n}{\partial T / \partial n}\right| .
$$

Furthermore, since pressure $p$, which is proportional to $\rho T$, in the boundary layer is approximately invariant along the normal direction $n$, we have $\rho(\partial T / \partial n)+T(\partial \rho / \partial n)=0$. Accordingly, the ratio of the two Knudsen numbers becomes

$$
\frac{\mathrm{Kn}_{D}}{\mathrm{Kn}_{T}} \approx 1,
$$

at a small distance from the wall. The same proof applies to $P$.

${ }^{1}$ G. A. Bird, Molecular Gas Dynamics and the Direct Simulation of Gas Flows (Oxford University Press, Oxford, 1994).

${ }^{2}$ G. V. Candler, I. Nompelis, and M.-C. Druguet, "Navier-Stokes predictions of hypersonic double-cone and cylinder-flare flow field," AIAA Paper 2001-1024 (2001).

${ }^{3}$ I. D. Boyd and W.-L. Wang, "Monte Carlo computations of hypersonic interacting flows," AIAA Paper 2001-1029 (2001).

${ }^{4}$ M. S. Holden, "Experimental database from CUBRC studies in hypersonic laminar and turbulent interacting flows including flowfield chemistry," RTO Code Validation of DSMC and Navier-Stokes Code Validation Studies, CUBRC Report, 2000.

${ }^{5}$ J. K. Harvey, M. S. Holden and T. P. Wadhams, "Code validation study of laminar shock-boundary layer and shock-shock interactions in hypersonic flow. Part B: Comparison with Navier-Stokes and DSMC solutions," AIAA Paper 2001-1031 (2001).

${ }^{6} \mathrm{G}$. A. Bird, "Breakdown of translational and rotational equilibrium in gaseous expansions," AIAA J. 8, 1998 (1970).

${ }^{7}$ I. D. Boyd, G. Chen, and G. V. Candler, "Predicting failure of the continuum fluid equations in transitional hypersonic flows," Phys. Fluids 7, 210 (1995).

${ }^{8}$ J. D. George and I. D. Boyd, "Simulation of nozzle plume flows using a combined CFD-DSMC approach," AIAA Paper 1999-3454 (1999).

${ }^{9}$ D. B. Hash and H. A. Hassan, "Assessment of schemes for coupling Monte Carlo and Navier-Stokes solution methods," J. Thermophys. Heat Transfer 10, 242 (1996)

${ }^{10}$ S. Y. Chou and D. Baganoff, "Kinetic flux-vector splitting for the NavierStokes equations," J. Comput. Phys. 130, 217 (1997).

${ }^{11}$ T. Lou, D. C. Dahlby, and D. Baganoff, "A numerical study comparing kinetic flux-vector splitting for the Navier-Stokes equations with a particle method," J. Comput. Phys. 145, 489 (1998).

${ }^{12}$ A. L. Garcia, J. B. Bell, W. Y. Crutchfield, and B. J. Alder, "Adaptive mesh and algorithm refinement using direct simulation Monte Carlo," J. Comput. Phys. 154, 134 (1999).

${ }^{13}$ T. I. Gombosi, Gaskinetic Theory (Cambridge University Press, Cambridge, 1994).

${ }^{14}$ F. G. Blottner, M. Johnson, and M. Ellis, "Chemically reacting viscous flow program for multi-component gas mixtures," Sandia Laboratories Report No. SC-RR-70-754, 1972.

${ }^{15}$ T. Gökçen and R. W. MacCormack, "Nonequilibrium effect for hypersonic transitional flows using continuum approach," AIAA Paper 19890461 (1989).

${ }^{16}$ S. Dietrich and I. D. Boyd, "Scalar and parallel optimized implementation of the direct simulation Monte Carlo method," J. Comput. Phys. 126, 328 (1996).

${ }^{17}$ K. Koura and H. Matsumoto, "Variable soft sphere molecular model for air species," Phys. Fluids A 4, 1083 (1992).

${ }^{18}$ I. D. Boyd, "Analysis of rotational nonequilibrium in standing shock waves of nitrogen," AIAA J. 28, 1997 (1990).

${ }^{19}$ P. Vijayakumar, Q. Sun, and I. D. Boyd, "Detailed models of vibrationaltranslational energy exchange for the direct simulation Monte Carlo method," Phys. Fluids 11, 2117 (1999).

${ }^{20}$ W.-L. Wang, I. D. Boyd, G. V. Candler, and I. Nompelis, "Particle and continuum computations of hypersonic flow over sharp and blunted cones," AIAA Paper 2001-2900 (2001).

${ }^{21}$ J. N. Moss, "DSMC computations for regions of shock-shock and shockboundary layer interaction," AIAA Paper 2001-1027 (2001). 